\title{
Proteomics in cardiovascular surgery
}

\author{
Peter Matt, MD, a,b Thierry Carrel, MD, ${ }^{a}$ Melanie White, $\mathrm{PhD}^{\mathrm{b}}$ Ivan Lefkovits, $\mathrm{PhD}^{\mathrm{a}}$ and Jennifer Van Eyk, $\mathrm{PhD}^{\mathrm{b}}$
}

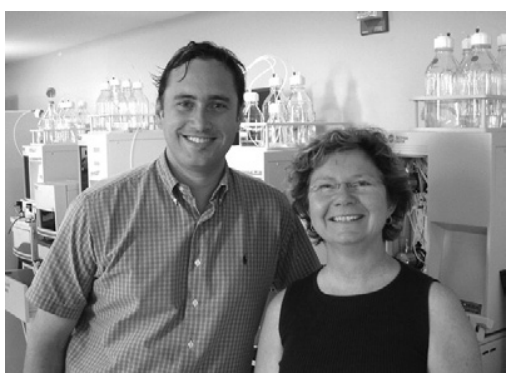

Drs Matt and Van Eyk

丹 Supplemental material is available online.
From the Division of Cardio-Thoracic Surgery, ${ }^{\text {a }}$ University Hospital Basel, Basel, Switzerland, and Johns Hopkins Proteomics Center, ${ }^{\mathrm{b}}$ Johns Hopkins University, Baltimore, Md.

Dr Matt is supported by the Swiss National Foundation, the Novartis Foundation, and the Hippocrate Foundation Basel for financial support. Dr Van Eyk is supported by grants from the National Heart, Lung, and Blood Institute Proteomic Initiative (contract N0-HV-28120) and the Daniel P. Ames Family Foundation.

Received for publication Aug 22, 2006; revisions received Aug 22, 2006; accepted for publication Sept 7, 2006.

Address for reprints: Peter Matt, MD, Johns Hopkins Proteomics Center, 5200 Eastern Ave, 607 MFL Bldg Center Tower, Baltimore, MS 21224 (E-mail: pmatt@uhbs.ch).

J Thorac Cardiovasc Surg 2007;133:210-4

$0022-5223 / \$ 32.00$

Copyright $\odot 2007$ by The American Association for Thoracic Surgery

doi:10.1016/j.jtcvs.2006.09.007
Proteomics describes, analogous to the term genomics, the study of the complete set of proteins present in a cell, organ, or organism at a given time. The genome tells us what could theoretically happen, whereas the proteome tells us what does happen. Therefore, a genomic-centered view of biologic processes is incomplete and does not describe what happens at the protein level. Proteomics is a relatively new methodology and is rapidly changing because of extensive advances in the underlying techniques. The core technologies of proteomics are 2-dimensional gel electrophoresis, liquid chromatography, and mass spectrometry. Proteomic approaches might help to close the gap between traditional pathophysiologic and more recent genomic studies, assisting our basic understanding of cardiovascular disease. The application of proteomics in cardiovascular medicine holds great promise. The analysis of tissue and plasma/serum specimens has the potential to provide unique information on the patient. Proteomics might therefore influence daily clinical practice, providing tools for diagnosis, defining the disease state, assessing of individual risk profiles, examining and/or screening of healthy relatives of patients, monitoring the course of the disease, determining the outcome, and setting up individual therapeutic strategies. Currently available clinical applications of proteomics are limited and focus mainly on cardiovascular biomarkers of chronic heart failure and myocardial ischemia. Larger clinical studies are required to test whether proteomics may have promising applications for clinical medicine. Cardiovascular surgeons should be aware of this increasingly pertinent and challenging field of science.

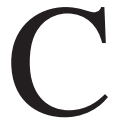
lassical cardiovascular research has involved histopathologic descriptions, pathophysiologic processes, identification of relevant receptors, analysis of signal transduction pathways, molecular gene mapping, and gene expression. Although the blueprint of all biologic functions is provided at the genomic level, their final manifestation occurs at the protein level. Therefore, a genomiccentered view of biologic processes is incomplete and does not describe what happens at the protein level. Analogous to genomics, the term proteomics describes the study of the complete set of proteins present in a cell, organ, or organism at a given time. ${ }^{1}$ The genome tells us what could theoretically happen, but the proteome tells us what does happen. It is the proteome that ultimately determines the phenotype. The concept of mapping the human proteome is more than 2 decades old. Rapid technologic advances in molecular biology, however, shifted the focus of basic researchers toward genomics for some years, culminating in the completion of the sequencing of the human genome. Microarrays and DNA chips are routinely used in cardiovascular biology and medicine nowadays. Proteomics will now help to close the gap between traditional pathophysiologic and more recent genomic studies, assisting our basic understanding of cardiovascular disease.

It is estimated that there are up to 10 times as many proteins as genes in human subjects. ${ }^{2}$ Not every gene is transcribed or translated into a single protein. Alternative splicing, numerous types of posttranslational modifications (eg, phosphorylation, glycosylation, and oxidation), and protein processing extend the number of protein species in a cell (Figure 1). Interactions between the modified proteins and the dynamics of protein expression under specific biologic circumstances lead to an 
Abbreviations and Acronyms

2-DE $=2$-dimensional gel electrophoresis

enormous molecular complexity, the resolution of which demands proteomic approaches. Protein-protein interactions might be a key to understanding basic molecular processes and how these are involved in and lead to the development of cardiovascular disease. Proteomics has the potential not only to revolutionize basic cardiovascular research but also to influence daily clinical practice, providing tools for diagnosis, defining the disease state, assessing individual risk profiles, examining and/or screening of healthy relatives of patients, monitoring the course of the disease, determining the outcome, and setting up individual therapeutic strategies. Proteomics itself is changing rapidly because of extensive advances in the underlying techniques.

Cardiovascular surgeons should be aware of this increasingly pertinent and challenging field of science. We describe below the basic methods of proteomics and how these are already or could be applied in clinical practice.

\section{Two-dimensional Gel Electrophoresis and Liquid Chromatography Techniques}

The first technology to be used in proteomics was 2dimensional gel electrophoresis (2-DE). It was developed independently in the laboratories of $\mathrm{O}^{\prime}$ Farrell $^{3}$ and $\mathrm{Klose}^{4}$ more than 2 decades ago. Proteins are amphoteric molecules that carry positive, negative, or zero net charge depending on their amino acid composition and the $\mathrm{pH}$ of their surroundings (molecular charge). In 2-DE, the first dimension of separation of proteins, known as isoelectric focusing, is based on the differences in their molecular charge. The second dimension then separates the proteins according to their molecular mass. ${ }^{5}$ The size separation is done in a polyacrylamide matrix in a sodium dodecylsulfate milieu (eg, sodium dodecylsulfate-polyacrylamide gel electrophoresis). Proteins can be visualized in the 2-dimensional gel by using different detection methods. The more common include Coomassie blue or silver staining (Figure 2), use of fluorescence dye (eg, Cy2, Cy3, and Cy5 dyes), radiolabeling, and immunodetection. ${ }^{6,7}$ Two-dimensional gels are evaluated and analyzed by using specialized software packages to compare 2-dimensional gel patterns and highlight differences between gel images.

Although 2-DE is a popular protein separation technique for proteomic analyses, other methods for separating and identifying proteins have been evolved. Many of these involve liquid chromatography techniques, which use solidand liquid-phase media to separate proteins or their peptide fragments according to specific biochemical properties, such as molecular mass, isoelectric point, or hydrophobicity. ${ }^{8}$ Some of these methods have advantages over 2-DE because they can be used on either native or denaturated proteins. Another separation technique, affinity chromatography, exploits the affinity of proteins for antibodies or specific target proteins. Current data suggest that using multiple technologies dramatically increases the number of detected proteins, especially those present in the sample at very low abundance.

\section{Mass Spectrometry}

Each molecular species of a protein is defined by its amino acid sequence, which is, at least in part, unique. Methods for establishing amino acid sequences by means of sequential cleavage of each consecutive $\mathrm{N}$-terminal amino acid were devised decades ago. ${ }^{5}$ When it became apparent that the mass of small peptides can be most accurately measured by means of mass spectrometry and the amino acid composition can be deduced from the mass data, considerable effort was invested in adopting methods of working with "true" polypeptides. Because the prerequisite for such analysis is conversion of the analyte into gas-phase ions, proteins must be cleaved into peptidic fragments and then converted into gas-phase ions and ejected to measure the time of flight. The measured masses of the resolved analytes are then compared with those calculated for the predicted peptides from the genomic and protein database, and the molecular identity of the analyzed protein is established. Mass spectrometers consist of 3 principal parts ${ }^{9-11}$ : an ionization source that converts molecules into gas-phase ions, a mass analyzer

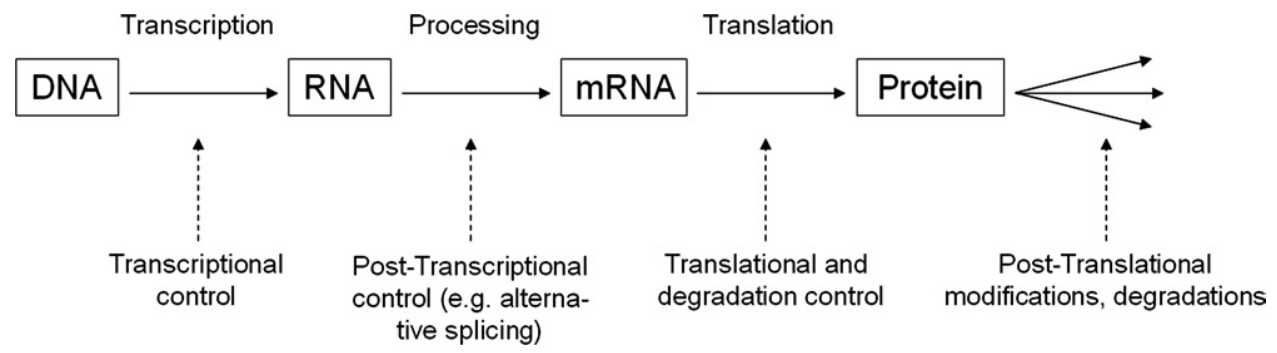

Figure 1. Outline of the relationship between the genome and proteome, showing the ways in which gene expression can be regulated or modified from transcription to posttranslation. 


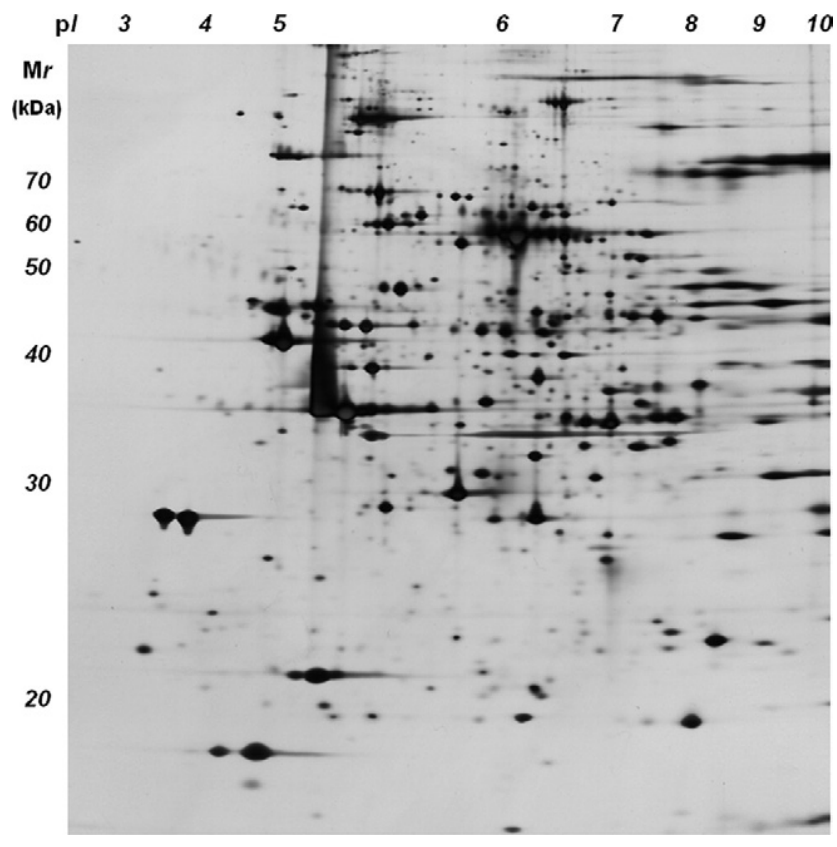

Figure 2. Two-dimensional gel pattern of a human left ventricular biopsy specimen. The patient subsequently underwent heart transplantation because of dilatative cardiomyopathy. The gel size measures $17 \times 17 \mathrm{~cm}$, the isoelectric point $(\mathrm{pl})$ range is 3 to 10, and the molecular mass (Mr) range is 15 to $100 \mathrm{kd}$. There are approximately 600 protein spots on the 2-dimensional gel, which can be further identified with mass spectrometry.

that separates individual components according to their mass-to-charge ratio (usually measuring the time of flight), and an ion detector that measures the magnitude of the current (as a function of time). Two ionization procedures are used in mass spectrometry. The first of these is matrixassisted laser desorption ionization, in which the peptides are deposited on an energy-absorbing crystalline matrix, and subsequently, the laser energy strikes the matrix and causes excitation of the matrix and ejection of the ions into the gas phase. The second procedure is electrospray ionization, in which the peptides in a solvent are induced by application of a potential to form a spray, the electrospray creates nanometer-size droplets, and subsequently the solvent is removed (by evaporation) and multiply charged ions are detected. These and other approaches yield highly precise estimates of the molecular mass of the peptide sample. Matrix-assisted laser desorption ionization is typically used in conjunction with time-of-flight mass analyzers, whereas further information is obtained by using tandem mass spectrometers, which produce a ladder of fragment ions that represent cleavage of amide bonds. Peptide molecular weight measurements predict amino acid composition, and peptide fragmentation information relates to the amino acid sequence. Both types of information can be correlated with protein sequences of databases. A single peptide mass, of course, is not unique to a specific protein, and thus a collection of peptides (from the same tryptic digest) has to provide the required match. A peptide spectrum, presented as plots of intensity versus mass-to-charge ratio, is obtained (Figure E1), which is a diagnostic identifier of the given protein sequence. Mass spectrometry sensitivity is improving rapidly, with routine detection limits currently at the femtomole $\left(10^{-15}\right)$ level.

\section{Proteomics in Cardiovascular Surgery}

Proteomic techniques have been mainly used in basic cardiovascular research. The focus has been on heart failure, in particular dilated cardiomyopathy. Animal models and transplanted human heart biopsy specimens have been investigated. The pioneering work of Dunn and colleagues and Jungblut and coworkers revealed more than 100 cardiacspecific proteins that are significantly altered in human dilatative cardiomyopathy tissue, with the majority of these proteins being less abundant in the diseased heart compared with control samples. ${ }^{12-15}$ If and how these proteins promote ventricular systolic dysfunction into manifest heart failure has yet to be evaluated. Most parts of the complex pathogenesis of human heart failure remain to be determined. In almost all forms of heart failure, the initial step is a compensatory myocardial adaptation, such as left ventricular hypertrophy in, for example, advanced heart disease. Ventricular remodeling can, if properly treated, still be reversed. Beyond this stage, however, an inadequate hypertrophy develops, with progressive exhaustion of myocardial energy resources, alterations in the cardiomyocytes, and changes in the extracellular matrix, eventually leading to terminal heart failure. Based on these considerations, the optimal time interval for a cardiovascular intervention, such as heart valve reconstruction or replacement in aortic or mitral valve disease, can be defined as the time point in the course of the disease at which all myocardial changes are still completely reversible. Clinical, echocardiographic, or hemodynamic parameters do not often determine precisely the transition from adequate to inadequate myocardial adaptation processes. The proteomic approach might provide an important contribution in determining the precise molecular pathways that lead to this specific point of no return in the development of human heart failure. This might allow the timing of cardiovascular surgical intervention to be optimized, leading at last to an improvement in patients' outcomes. With this objective, most clinical applications of proteomics have focused on serologic biomarkers.

A biomarker can be defined as a measurable cell, protein, peptide, gene, or metabolic product that represents biologic processes in an organism at a given time. ${ }^{16,17}$ Biomarkers delineate variances from normal biology and either appear for the first time (newly expressed) or are increased beyond 
the normal range in response to 1 or more stimuli or pathologic events. One or several biomarkers might be an indicator for the disease, the disease state, or the risk that the disease will progress; the biomarker or biomarkers might also indicate the prognosis, the likely response to a therapeutic (eg, surgical) intervention, or both. It is important to realize that, unlike cells or tissues, serum or plasma does not have a genome, and therefore proteomic techniques are the only option for studying biomarkers in the blood. Detailed lists of available cardiovascular biomarkers have recently been published by Vasan ${ }^{18}$ and Maisel and coworkers. ${ }^{19}$ However, few biomarkers are routinely used in cardiovascular medicine. Brain natriuretic peptide is used for diagnostic reasons and to adapt medical therapies in symptomatic patients with heart failure. ${ }^{18-20}$ Brain natriuretic peptide holds great promise, but its specificity and sensitivity are limited and are influenced by a variety of factors. Heart transplantation has been the gold standard for patients with severe chronic heart failure. Nevertheless, because of the shortage of donor organs, only a small number of patients receive this therapeutic option. Long-term outcome after heart transplantation mainly depends on the development of cardiac allograft vasculopathy and rejection. The right ventricular endomyocardial biopsy serves as a source for diagnostic analysis, although this is an invasive procedure.

Proteomic approaches could serve as a powerful and noninvasive tool providing insights into the molecular myocardial mechanisms associated with the immune response and graft function. ${ }^{1,21}$ Mehra and associates ${ }^{22}$ recently emphasized the need for such peripheral markers for late cardiac allograft monitoring. Our research group and others are attempting to generate a proteomic approach using peripheral blood samples, with the aim of avoiding biopsy and defining proteomic patterns that can be used to generate a score to indicate chronic and acute rejection. Shortage of donor organs has led to increased use of mechanical left ventricular assist devices. These devices have become a reliable tool for stabilizing the condition of medically refractory patients with end-stage heart failure awaiting transplantation. Left ventricular assist devices support triggers within the failing myocardium, a multitude of adaptational mechanisms that lead, in certain circumstances, to improved cardiac function and decreases in heart size and the concentration of plasma neurohormones and cytokines. Such devices might also lead to normalization of the expression of cell-surface receptors, inhibition of apoptotic pathways, and decreased ventricular expression of natriuretic peptides and tumor necrosis factor $\alpha$. It has been suggested that in some instances mechanical unloading provides enough improvement that heart transplantation is no longer necessary. Predictors of such cardiac reverse remodeling processes are not available at the moment, but proteomic techniques might provide a suitable diagnostic or monitoring tool.
Biomarkers for upcoming events, such as myocardial ischemia/infarction, heart failure, aortic aneurysm formation, or acute aortic dissection are still lacking. MateosCaceres and colleagues ${ }^{23}$ recently showed how proteomic techniques (in serologic samples) could be used to differentiate between patients with unstable angina and those with acute myocardial infarction. Although their results are preliminary, the proteomic patterns showed many differences in protein expression between the patient groups, in particular in $\alpha_{1}$-antitrypsin isoforms, fibrinogen $\gamma$-chain isoforms, and apolipoprotein A-I isoforms. McDonough and Van $\mathrm{Eyk}^{24}$ also recently revealed that determination of troponin isoforms might help in differentiating acute myocardial infarction, pulmonary lung embolism, and acute aortic dissection, which would be of great value in daily clinical practice. Proteomic techniques have also been used to investigate vascular disease, mainly focusing on the development of arteriosclerosis. The proteome and secretome (which corresponds to the proteins secreted by a cell at a certain time) of human arterial smooth muscle cells, which play a crucial role in vascular diseases, has recently been published by Dupont and associates. ${ }^{25}$ With regard to clinical applications, some biomarkers for vascular inflammation, lipid deposition, plaque rupture, and remodeling processes have been demonstrated. However, the sensitivities and specificities of these markers (eg, C-reactive protein, interleukin 6, interleukin 10, interleukin 18, and heat shock protein 27) are low, and therefore it is unlikely that they will be clinically useful. ${ }^{26-28}$

For ascending aortic aneurysm formation, upcoming aortic rupture, or acute aortic dissection, biomarkers are currently lacking. Our research group, a collaboration between the Johns Hopkins University, Baltimore, and the University Hospital Basel, is focusing on markers for appearance or progression of aortic root dilation in Marfan syndrome and bicuspid aortic valve, as well as anuloaortic ectasia with tricuspid aortic valve and the development of acute aortic dissection. We expect to reveal new insights into the underlying molecular mechanism leading to aneurysm formation and in consequence to develop diagnostic markers that will simplify patient monitoring and eventually optimize patient management. Feezor and coworkers ${ }^{29}$ recently found that the proteomic pattern in preoperatively collected serum samples could discriminate between patients who had a multisystem organ dysfunction syndrome after thoracoabdominal aortic aneurysm repair and those who did not. The authors used proteomic and genomic techniques together to define the determinants of outcome. This work confirms that proteomic and genomic methods are not in competition but are complementary to each other. The availability of all biomarkers (the existing ones and those to be discovered) will not only improve our understanding 
of cardiovascular diseases, but it will provide a solid basis for an individualized patient management.

\section{How Can the Cardiovascular Surgeon Exploit Proteomics?}

The transfer of proteomics research from the observational bedside to the laboratory might provide important new insights in cardiovascular biology and medicine. Therefore, cardiovascular surgeons should be aware of this valuable, challenging, and rapidly changing field. A surgeon can play an important role in defining the clinical question, in experimental design and organization, and in interpreting the data. ${ }^{30}$ However, the surgeon will need a basic understanding of proteomic principles and techniques to interact competently with the basic scientist. ${ }^{31}$ As part of the research team, the surgeon has regular contact with the patient, collects the clinical data, collects samples in the operating room, and can focus the basic scientist on the most clinically relevant problems. The technical challenges in proteomics demand an interdisciplinary approach. This requires mutual appreciation and close collaboration between the clinician and the basic scientist.

The application of proteomics in cardiovascular medicine holds great promise. The analysis of tissue and plasma/ serum specimens has the potential to provide unique information about the patient and the underlying disease. Larger clinical studies are required to test whether proteomic approaches are applicable in clinics.

\section{References}

1. Zerkowski HR, Grussenmeyer T, Matt P, Grapow M, Engelhardt S, Lefkovits I. Proteomics strategies in cardiovascular research. J Proteome Res. 2004;3:200-8.

2. Loscalzo J. Proteomics in cardiovascular biology and medicine. Circulation. 2003;108:380-3.

3. O'Farrell PH. High resolution two-dimensional electrophoresis of proteins. J Biol Chem. 1975;250:4007-21.

4. Klose J. Protein mapping by combined isoelectric focusing and electrophoresis of mouse tissues. A novel approach to testing for induced point mutations in mammals. Humangenetik. 1975;26:231-43.

5. Lefkovits I, Grussenmeyer T, Matt P, Grapow M, Lefkovits M, Zerkowski HR. Proteomics in clinical research: perspectives and expectations. In: Faulus A, editor. Immunogenomics and human disease. London: Johns Wiley and Sons; 2005.

6. Gorg A, Obermaier C, Boguth G, Harder A, Scheibe B, Wildgruber R, et al. The current state of two-dimensional electrophoresis with immobilized pH gradients. Electrophoresis. 2000;21:1037-53.

7. Gygi SP, Corthals GL, Zhang Y, Rochon Y, Aebersold R. Evaluation of two-dimensional gel electrophoresis-based proteome analysis technology. Proc Natl Acad Sci U S A. 2000;97:9390-5.

8. Opiteck GJ, Ramirez SM, Jorgenson JW, Moseley MA 3rd. Comprehensive two-dimensional high-performance liquid chromatography for the isolation of overexpressed proteins and proteome mapping. Anal Biochem. 1998;258:349-61.
9. Quadroni M, James P. Proteomics and automation. Electrophoresis. 1999;20:664-77.

10. Yates JR 3rd. Mass spectrometry. From genomics to proteomics. Trends Genet. 2000;16:5-8.

11. Jungblut $\mathrm{P}$, Thiede B. Protein identification from 2-DE gels by MALDI mass spectrometry. Mass Spectrom Rev. 1997;16:145-62.

12. Knecht M, Regitz-Zagrosek V, Pleissner KP, Jungblut P, Steffen C, Hildebrandt A, et al. Characterization of myocardial protein composition in dilated cardiomyopathy by two-dimensional gel electrophoresis. Eur Heart J. 1994;15(suppl D):37-44.

13. Knecht M, Regitz-Zagrosek V, Pleissner KP, Emig S, Jungblut P, Hildebrandt A, et al. Dilated cardiomyopathy: computer-assisted analysis of endomyocardial biopsy protein patterns by two-dimensional gel electrophoresis. Eur J Clin Chem Clin Biochem. 1994;32:615-24.

14. Jungblut P, Otto A, Zeindl-Eberhart E, Plessner KP, Knecht M, Regitz-Zagrosek V, et al. Protein composition of the human heart: the construction of a myocardial two-dimensional electrophoresis database. Electrophoresis. 1994;15:685-707.

15. McGregor E, Dunn MJ. Proteomics of the heart: unraveling disease. Circ Res. 2006;98:309-21.

16. Becker RC. Proteomics, metabolomics and circulating endothelial progenitor cells in acute coronary syndromes. J Thromb Thrombolysis. 2006;21:203-6.

17. Fu Q, Van Eyk JE. Proteomics and heart disease: identifying biomarkers of clinical utility. Expert Rev Proteomics. 2006;3:237-49.

18. Vasan RS. Biomarkers of cardiovascular disease: molecular basis and practical considerations. Circulation. 2006;113:2335-62.

19. Maisel AS, Bhalla V, Braunwald E. Cardiac biomarkers: a contemporary status report. Nat Clin Pract Cardiovasc Med. 2006;3:24-34.

20. Mueller C, Scholer A, Laule-Kilian K, Martina B, Schindler C, Buser $\mathrm{P}$, et al. Use of B-type natriuretic peptide in the evaluation and management of acute dyspnea. $N$ Engl J Med. 2004;350:647-54.

21. Mehra MR, Benza R, Deng MC, Russell S, Webber S. Surrogate markers for late cardiac allograft survival. Am J Transplant. 2004;4: 1184-91.

22. Mehra MR. The emergence of genomic and proteomic biomarkers in heart transplantation. J Heart Lung Transplant. 2005;24(suppl): S213-8.

23. Mateos-Caceres PJ, Garcia-Mendez A, Lopez Farre A, Macaya C, Nunez A, Gomez J, et al. Proteomic analysis of plasma from patients during an acute coronary syndrome. $\mathrm{J} \mathrm{Am} \mathrm{Coll} \mathrm{Cardiol.}$ 2004;44:1578-83.

24. McDonough JL, Van Eyk JE. Developing the next generation of cardiac markers: disease-induced modifications of troponin I. Prog Cardiovasc Dis. 2004;47:207-16.

25. Dupont A, Corseaux D, Dekeyzer O, Drobecq H, Guihot AL, Susen S, et al. The proteome and secretome of human arterial smooth muscle cells. Proteomics. 2005;5:585-96.

26. Vivanco F, Martin-Ventura JL, Duran MC, Barderas MG, BlancoColio L, Darde VM, et al. Quest for novel cardiovascular biomarkers by proteomic analysis. J Proteome Res. 2005;4:1181-91.

27. Lipinski MJ, Fuster V, Fisher EA, Fayad ZA. Technology insight: targeting of biological molecules for evaluation of high-risk atherosclerotic plaques with magnetic resonance imaging. Nat Clin Pract Cardiovasc Med. 2004;1:48-55.

28. Blanco-Colio LM, Martin-Ventura JL, Vivanco F, Michel JB, Meilhac O, Egido J. Biology of atherosclerotic plaques: what we are learning from proteomic analysis. Cardiovasc Res. 2006;72:18-29.

29. Feezor RJ, Baker HV, Xiao W, Lee A, Huber TS, Mindrinos M, et al Genomic and proteomic determinants of outcome in patients undergoing thoracoabdominal aortic aneurysm repair. J Immunol. 2004;172: 7103-9.

30. Carrel T. The relationship between surgeon and basic scientist. Transpl Immunol. 2002;9:331-7.

31. Roblick UJ, Auer G. Proteomics and clinical surgery. Br J Surg. 2005;92:1464-5. 


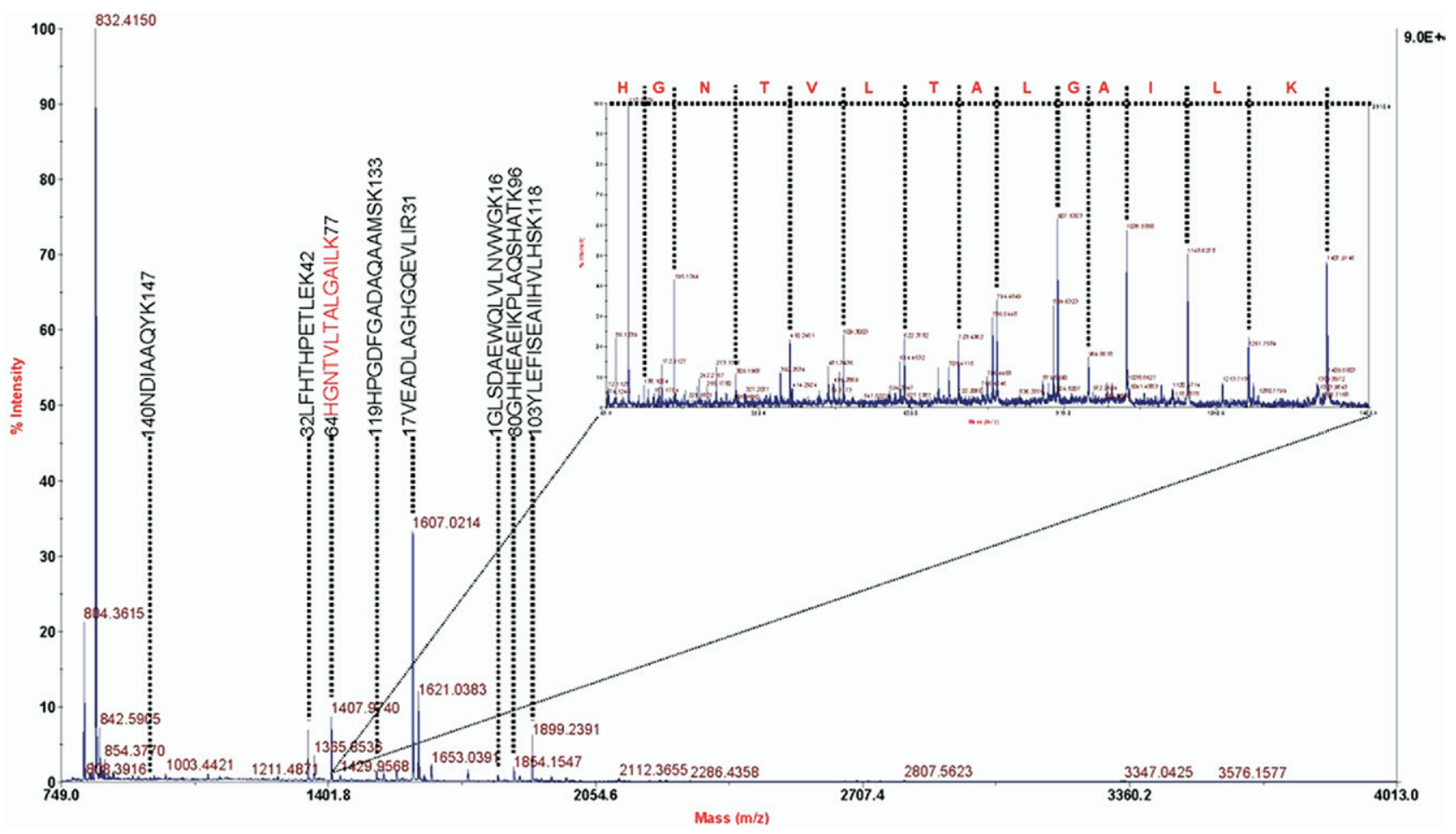

Figure E1. Peptides resulting from tryptic digestion of a protein spot resolved by means of 2-dimensional gel electrophoresis are analyzed by using mass spectrometry. The resulting mass spectra are presented as a plot of signal intensity versus mass-to-charge ratio $(\mathrm{m} / \mathrm{z})$. The peptide corresponding to an $\mathrm{m} / \mathrm{z}$ ratio of 1407.9 was selected for further analysis (inset). Fragmentation of peptide $1407.9 \mathrm{~m} / \mathrm{z}$ by means of mass spectrometry allowed for amino acid sequence deduction (as denoted in red letters). Using the combination of matrix-assisted laser desorption ionization-time-of-flight analysis and time-of-flight/time-of-flight analysis, $72.6 \%$ of the protein sequence was covered, and the protein was finally identified as myoglobin (isoelectric point, 6.67; molecular mass, 17.1 kd). 